\title{
Enrichment of carbon recovery of high ash coal fines using air fluidized vibratory deck separator
}

\author{
Laxmikanta Sahu ${ }^{1} \cdot$ Shobhana Dey ${ }^{2}$
}

Received: 2 February 2017/Revised: 17 May 2017/ Accepted: 24 June 2017 / Published online: 5 July 2017

(C) The Author(s) 2017. This article is an open access publication

\begin{abstract}
Coal is primarily beneficiated by wet gravity methods. The wet processing of coal is an efficient practice. However, it introduces the moisture in the range of $6 \%-15 \%$, depending upon the size of coal which is as detrimental as ash content to the heating value of coal. Dry beneficiation of coal fines was carried out using an air fluidized vibrating table in which the coal particles get separated from the heavier mineral particles as a result of horizontal and vertical stratification. Two level factorial design matrix was used to optimize and assess the interactive effects of the operational parameters of a pneumatic table viz. deck eccentric, side tilt and air flow rate on the clean coal yield and its ash content. Double stage processing was found to be more effective for reducing the ash content of the clean coal. Initial stage of processing at a higher ash level generates a reject of high ash with low combustibles. Cleaning of the rougher concentrate at $34 \%-35 \%$ ash level shows significant improvement in the organic efficiency $(88.6 \%)$ and useful heat value of clean coal $(15690 \mathrm{~kJ} / \mathrm{kg})$. The performance of air fluidized vibrating deck was measured by $E_{\mathrm{p}}$ value which is 0.18 .
\end{abstract}

Keywords Air flow rate $\cdot$ Deck eccentric $\cdot$ Segregation of particles $\cdot$ Separation efficiency

\section{Introduction}

The resources of coal in India are about 301.56 billion tonnes which are much higher compared to oil and gas (Geological survey of India (GSI) 2014). Out of total reserve, 266.00 billion tones are the proven reserve of noncoking coal. This indicates that about $88 \%$ is the noncoking coal. The coal contributes to about $55 \%$ of the commercial energy consumption as compared to $27 \%$ world average and more than $65 \%$ of electricity generation capacities are coal based. India is the third largest coal producing country after China and USA. The quality of the

Shobhana Dey

sd@nmlindia.org; shobhanade@gmail.com

1 Department of Fuel and Mineral Engineering, Indian Institute of Technology (Indian School of Mines), Dhanbad, Jharkhand 826004, India

2 Mineral Processing Division, CSIR-National Metallurgical Laboratory, Jamshedpur, Jharkhand 831007, India
Indian coal is inferior because of drift origin and causes dissemination of the mineral matter.

An Indian power plant of high-ash coals is energy intensive, causing shortages of rail cars and trucks. The existing boilers are designed for treating the coal having ash of $25 \%-35 \%$. The beneficiated thermal coal fines can increase the thermal efficiencies of the boilers. Coal is currently beneficiated by wet method through gravity and flotation techniques. Moisture retained in the washed products is about $6 \%-15 \%$, depending upon the feed size. Removal of moisture requires significant amount of energy and subsequently increases the operating cost. Dry processing can be used in the area of arid region. It is more economic than wet cleaning as dewatering systems are not involved. The cost of cleaning fine coal by wet method is estimated at three to six times the cost of cleaning coarser coal, the range being dependent on particle size. So it can be eliminated by dry cleaning of coal. It is also examined that product of dry cleaning has higher thermal efficiency than that of wet cleaning. Indian Government has made a rule that coal transported from a distance of $1000 \mathrm{~km}$ or 
more for the thermal power plant or burnt in a sensitive area must be beneficiated to reduce the ash level to $34 \%$ (Ministry of Environment and Forest 1998).

Dry cleaning of coal has attracted the attention of the researchers worldwide because of the obvious economic advantages it offers. Studies have been carried out in most of the major coal producing countries to evaluate the performance of the dry coal cleaning units. Some researchers have worked on air dense-medium fluidised bed for large sized coal where fine magnetite powder is used. Test work has been carried out at different size spectra of coal.

Air tables are found to be more useable than any other dry gravity concentrator particularly in the food industry as they are originally developed for seed separation (Burt 1984). In early 1916, dry beneficiation of coal fines was introduced in United States (Lockhart 1984). The pneumatic density separators were widely practiced between 1930 and 1960. Several researchers found that dry beneficiation of coarse coals are found to be effective in reducing the ash in clean coal by different techniques ( $\mathrm{Li}$ et al. 2011; Yang et al. 2013). In air fluidization technique, autogeneous medium is produced by mixture of air and fine coal power which behaves as a separating density. The dry processing of coal fines was carried out by Patil and Parekh (2011) and Shobhana et al. (2015) for reducing the ash content in the clean coal by air fluidization technique. Air tables are also being used for the dry beneficiation of beach sand for separation of heavy minerals (Gupta et al. 2012), enrichment of tungsten values (Honaker et al. 2008). The pneumatic table was also used for dry separation of mixture of plastics (Dodbiba et al. 2005). Zhao et al. studied on degree of segregation of fine lignite in a vibrated gas-fluidized bed (Zhao et al. 2015). Theoretical considerations show that effective separation in a table can be achieved when the time of free fall could be minimized (Osborne 1988; Haider and Levenspiel 1989; Zhao et al. 2002). The separation of fine particles in air is difficult due to low differences in settling velocity. However, the modern techniques assist to increase the efficiency of separation by introducing other parameters, like transverse oscillation, slanting the deck in longitudinal and transverse direction which increases the flow of the particles according to the desired grade.

In India the environmental regulations make it imperative for the thermal power plants to charge the coal having $34 \%$ ash in the boilers. Therefore, efforts have been made to produce a product that satisfies the ash requirement. In the present investigation, dry beneficiation of high ash coal fines has been studied for reduction of ash in clean coal, enrichment of carbon values and also for understanding the separation behavior. Interactive effects of process variables of an air fluidized vibratory table on the responses have been highlighted.

\section{Material and method}

\subsection{Material}

Thermal coal of G-grade collected from Talcher coal fieldwas used for the study. Characterization of the coal sample was carried out at $-1 \mathrm{~mm}$. Coal petrography was done through Advanced Polarising Microscope (Leica DM 4500, Germany) following BIS procedure (IS 9127-5) on polished mounts. It appears un-banded durainous in nature. Mineral matters are present in association with different maceral groups (Fig. 1a, b). On visual basis, mineral matters constituted $60.8 \%$. Mineral matter occurs in dark colour either as cavity filling or in disseminated form. This component is rich in clay minerals, silica (quartz) and carbonates (siderite). Among the macerals, vitrinite group of macerals are dominating followed by the inertinite and liptinite macerals. Figure 1 also shows that presence of clear bands of mineral matter, vitrinite and inertinite assist in recovering of clean coal. However, there is also fine dissemination of mineral matter with the macerals which indicates the inter locking of coaly matters. The distribution of petrographic constituents is shown in Table 1 .

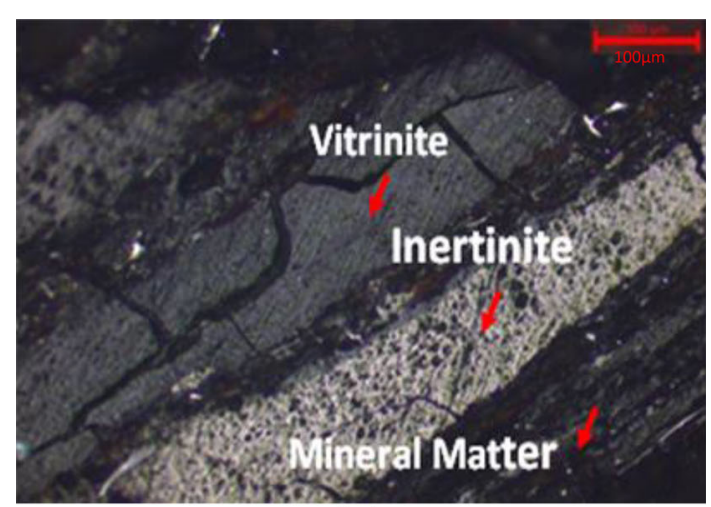

(a)

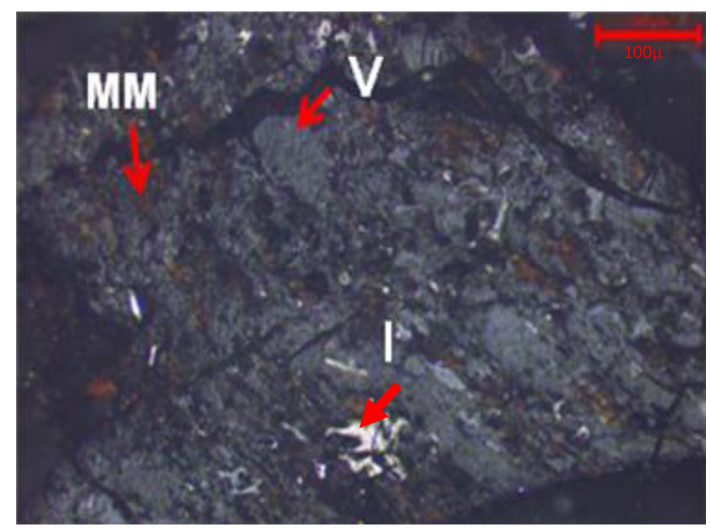

(b)

Fig. 1 Microphotographs of coal sample 
Table 1 Petrography analysis of coal sample

\begin{tabular}{lc}
\hline Macerals & Volume (\%) \\
\hline Vitrinite & 24.4 \\
Inertinite & 12.5 \\
Liptinite & 2.3 \\
Mineral matter & 60.8
\end{tabular}

Table 2 Proximate analysis of coal sample

\begin{tabular}{lllll}
\hline Moisture (\%) & Ash (\%) & VM (\%) & FC (\%) & Heat value $(\mathrm{kJ} / \mathrm{kg})$ \\
\hline 1.9 & 54.5 & 20.2 & 23.4 & 4979 \\
\hline
\end{tabular}

The proximate analysis (Table 2) of coal sample was carried out as per the IS standard (IS 1350-1) using Coal analyzer. The heat value of the coal sample is quite low as $4979 \mathrm{~kJ} / \mathrm{kg}$ because of high ash content of $54.5 \%$ and this corroborate the petrographic analysis of the mineral matter in the coal sample.

From the characterization study of the coal sample, it was found that beneficiation would be effective at finer size as the coal sample is adequately liberated. The sample was stage crushed to $1 \mathrm{~mm}$ and $-150 \mu \mathrm{m}$ fraction was discarded. The $-1+0.15 \mathrm{~mm}$ fraction was treated in a pneumatic table. Characterization of the feed material was carried out in terms of size distribution, ash and heat values of each fraction (Fig. 2). The high ash in the fractions reflects that recovery of clean coal at low specific gravity is difficult to achieve. It also signifies to extend the washability study of the sample up to high density of the medium, varying from 1.4 to 2.2 . The useful heat value in Fig. 2 indicates that the fractions $-500+300$ and $-300+150 \mu \mathrm{m}$ possess higher value compared to other fractions.

\subsection{Methods}

The coal sample $(-1+0.15 \mathrm{~mm})$ was subjected to dry beneficiation using a vibratory-pneumatic deck. The separator consists of rectangular porous deck below which an air chamber. The deck is connected to an eccentric mechanism comprising of variable speed drive which triggers the required oscillation in the deck that imparts vibratory motion to the particles. As a result segregation of the particles takes place based on their densities. The deck can be slanted transversely and longitudinally. There are five process parameters, namely feed rate, deck eccentric, transverse angle, longitudinal angle and air flow rate. In present study, three variables, viz, deck eccentric, air flow rate and transverse angle were selected as operational parameters and the feed rate $(90 \mathrm{~kg} / \mathrm{h})$ and longitudinal angle $(78 \mathrm{~mm})$ were kept constant throughout the experiments. The factorial design of experiments was followed to conduct the tests for establishing the relationship between the process parameters and the response functions of recovery of combustibles and ash of clean coal. The sample was fed across the width of the deck approximately at the centre point (Fig. 3). Longitudinal angle of the deck is related to the residence time and provides the time for the

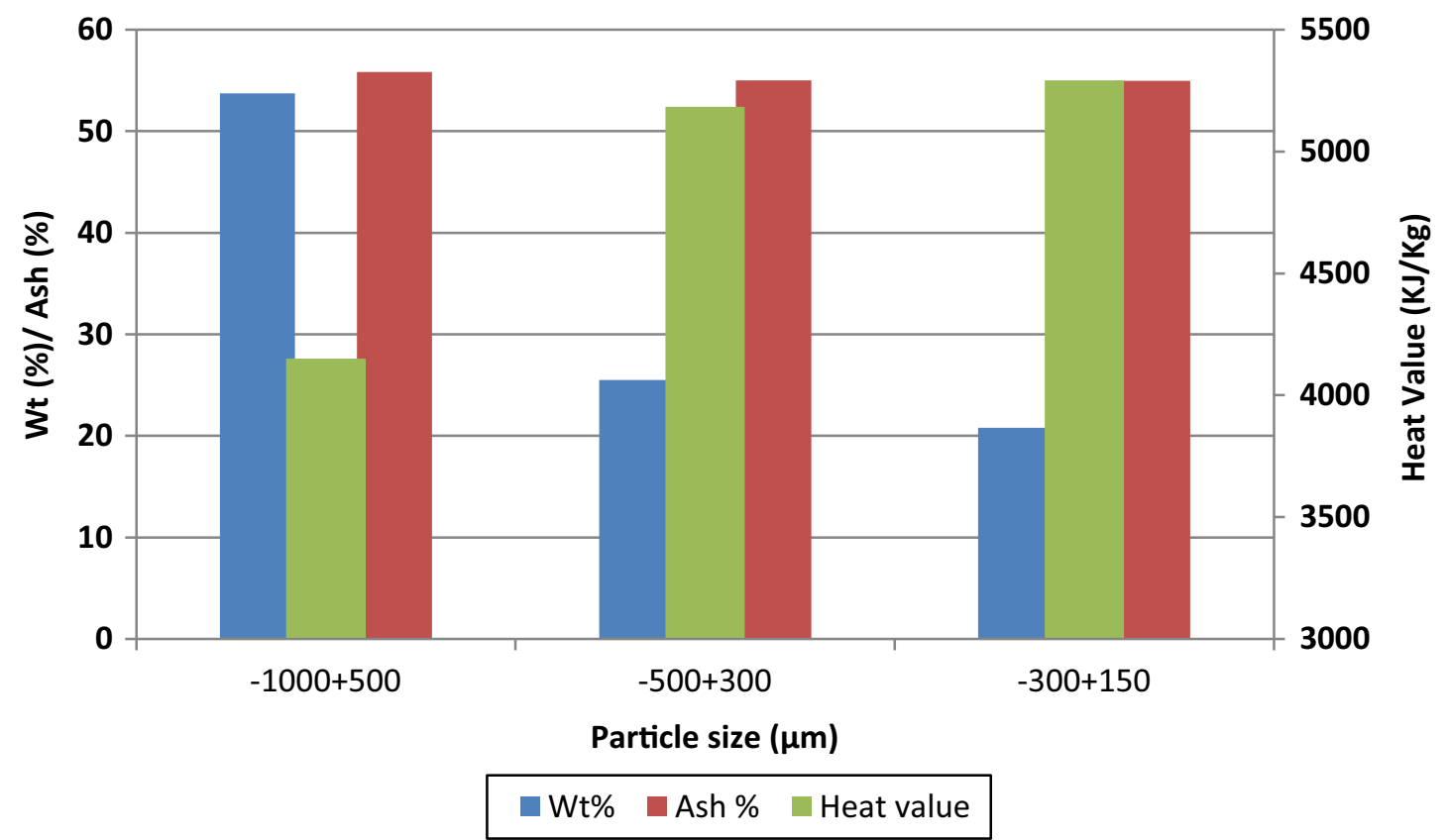

Fig. 2 Characterization of feed sample in terms of particle size distribution and heat value 


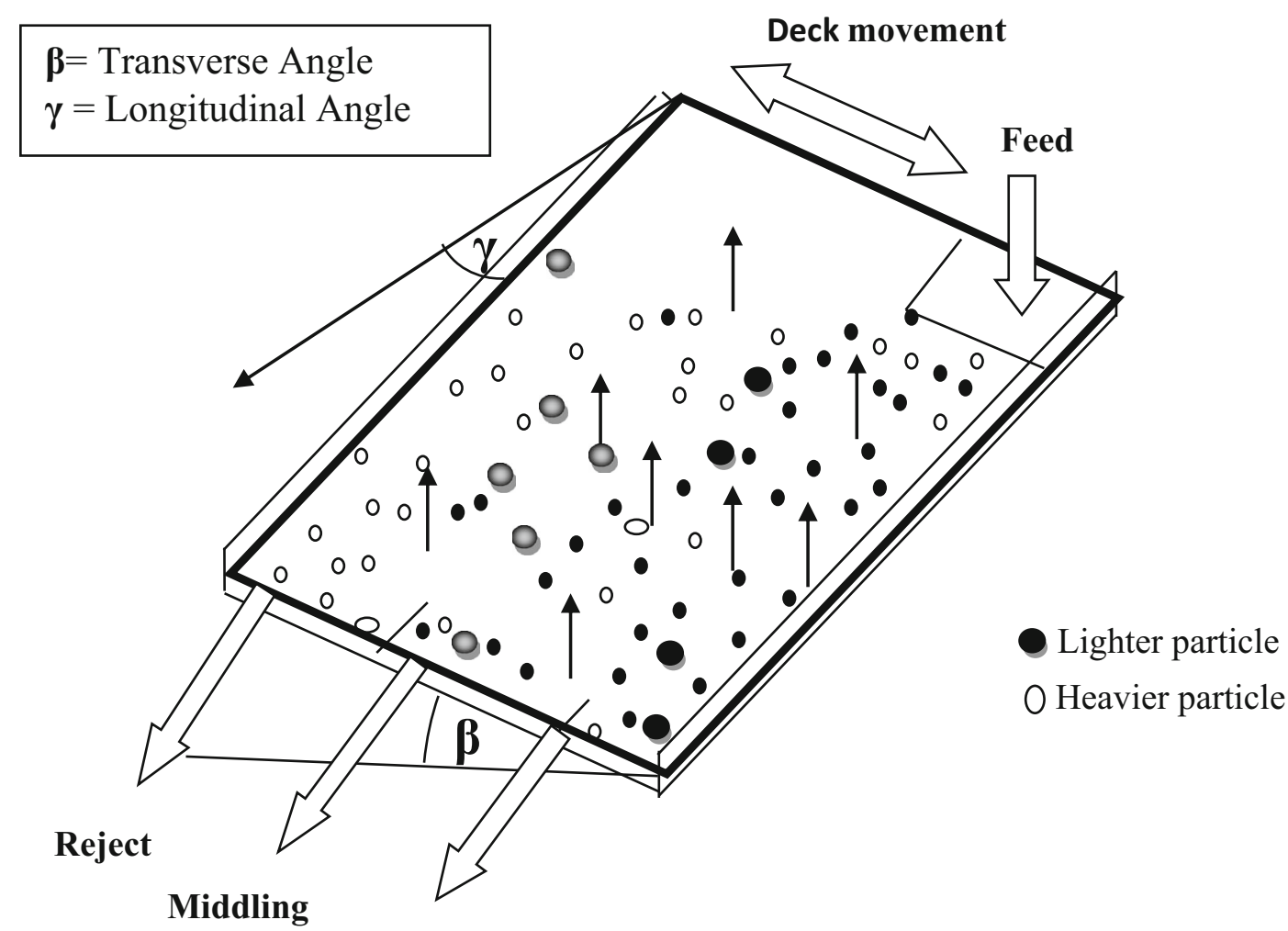

Light

Fig. 3 Schematic diagram of air fluidized vibratory deck separator

feed to expose for separation on the deck. The upward flow of air causes stratification of the bed. The heavier particles gravitate through the bed, come in contact with the deck and are moved up the deck surface due to the eccentric reciprocating motion. The lighter coal particles tend to float and move to the top of the bed and pushed down the deck by new feed material and resultant gravitational force. The deck eccentric pushes the heavier particles sideways towards the higher end of the deck. The side tilt governs the quality of separation. The dry beneficiation was also carried out in two stages. The first stage was targeted to generate a tailing with high ash content (about 80\%). The middling and light streams of the first stage were combined together for the second stage processing under different conditions for producing a clean coal at $34 \%-36 \%$ ash level.

\subsection{Process variables of vibratory table}

In this investigation, three operational variables including deck eccentric (DE), air flow rate (AFR) and transverse angle (SA) are taken into consideration. Three variables at two level factorial design of experiments were followed. There is an upper and lower limit of the range with a base level (average value of upper and lower values) for each process variable. The levels of different process variables are given in Table 3 .
Table 3 Process variables and their levels

\begin{tabular}{lllll}
\hline Parameters & Units & \multicolumn{2}{l}{ Levels } & \\
\cline { 3 - 5 } & & -1 & 0 & +1 \\
\hline Deck eccentric & $\mathrm{Hz}$ & 19 & 20 & 21 \\
Air flow rate & $\mathrm{rpm}$ & 32 & 41 & 50 \\
Side angle & $\mathrm{mm}$ & 40 & 50 & 60 \\
\hline
\end{tabular}

\section{Results and discussion}

Washability study of the coal sample and dry beneficiation carried out using an air fluidized vibratory deck are described below.

\subsection{Washing amenability of coal sample}

The washability study of coal sample conducted by using medium of varied specific gravities shows that at a specific gravity of 2.0, yield of clean coal is $40.4 \%$ at $24.3 \%$ ash level with recovery of combustibles $67.7 \%$ (Fig. 4). The amount of reject (sink) produced at this separation density is $59.6 \%$ with combustibles of $32.3 \%$ and $75.7 \%$ ash. Increasing the separating density to 2.1 , yield of clean coal becomes $53.2 \%$ with $77.7 \%$ combustibles and $34 \%$ ash. 


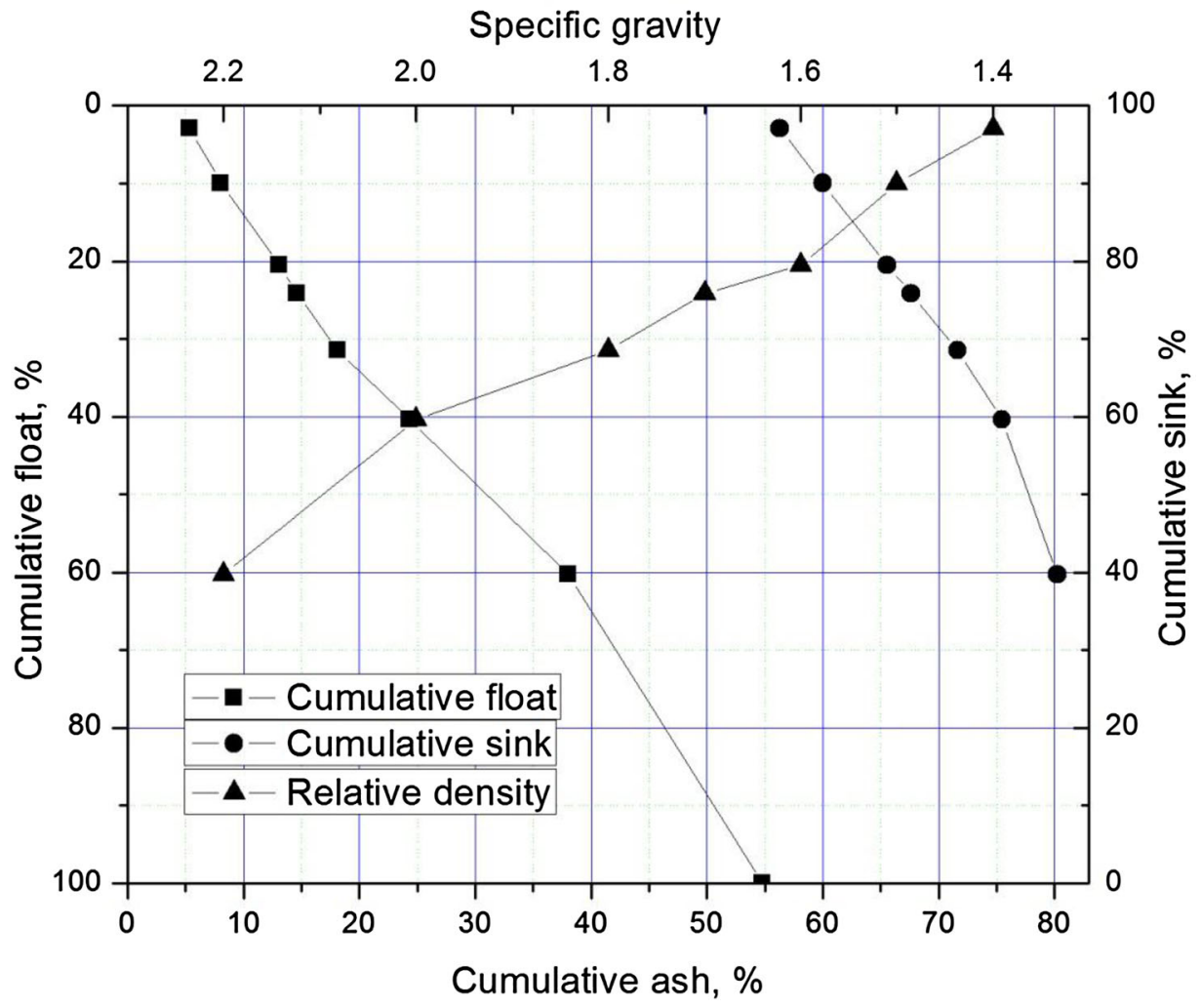

Fig. 4 Washability analysis of feed sample

The combustible matter in reject stream reduces to $22.3 \%$ with $78.5 \%$ ash. Further increase of the specific gravity to 2.15 , combustible matter in the concentrate could be significantly improved to $82.6 \%$ at $38 \%$ ash level and the same in the reject drastically reduces to $17.4 \%$ (Table 4 ). The coal desired for thermal power plant should be in the range of $34 \%-36 \%$ ash which was the objective to achieve from this study. The washability study also shows that Near Gravity Material (NGM) varies from $19.8 \%$ to $24.7 \%$. At higher separating density, NGM reduces which reveals that misplacement of the carbon values is less.

\subsection{Dry beneficiation}

Dry separation of high ash fine coal using an air fluidized vibratory separator under different conditions was carried out. The results are shown in Fig. 5. It shows that separation characteristics are dependent on the process parameters. Analyzing the experimental data of Air table using a Design Software version 8 (Design Expert), regression Eqs. (1) and (2) for the responses of yield and ash were derived. The optimal conditions are predicted by the analysis of variance (ANOVA). Predicted conditions were validated by conducting the tests. The experimental values are found to be good agreement with the predicted values. The effects of the variables on responses were described below. Regression equations for responses

$$
\begin{aligned}
\text { Yield } \%= & 66.58-4.94 \mathrm{DE}+1.54 \mathrm{AFR}+1.43 \mathrm{SA} \\
& -0.45 \mathrm{DE} * \mathrm{AFR}-0.094 \mathrm{DE} * \mathrm{SA} \\
\text { Ash } \%= & 14.49-1.04 * \mathrm{DE}+0.5 * \mathrm{AFR}+0.091 * \mathrm{SA} \\
& -0.18 * \mathrm{DE} * \mathrm{AFR}-0.16 * \mathrm{AFR} * \mathrm{SA}
\end{aligned}
$$

\begin{tabular}{|c|c|c|c|c|c|c|c|}
\hline \multirow[t]{2}{*}{ Ash level (\%) } & \multirow[t]{2}{*}{ Cut density (gm/cc) } & \multicolumn{2}{|c|}{ Clean coal $(\%)$} & \multicolumn{3}{|c|}{ Reject $(\%)$} & \multirow[t]{2}{*}{$\mathrm{NGM} \pm 0.1$} \\
\hline & & Yield & Rec. comb & Yield & Ash & Rec. comb & \\
\hline 24.3 & 2.0 & 40.4 & 67.7 & 59.6 & 75.7 & 32.3 & 23.22 \\
\hline 34.0 & 2.1 & 53.2 & 77.7 & 46.8 & 78.5 & 22.3 & 24.70 \\
\hline 37.0 & 2.15 & 60.2 & 82.6 & 39.8 & 80.3 & 17.4 & 19.84 \\
\hline
\end{tabular}

Table 4 Washability results at different ash levels 


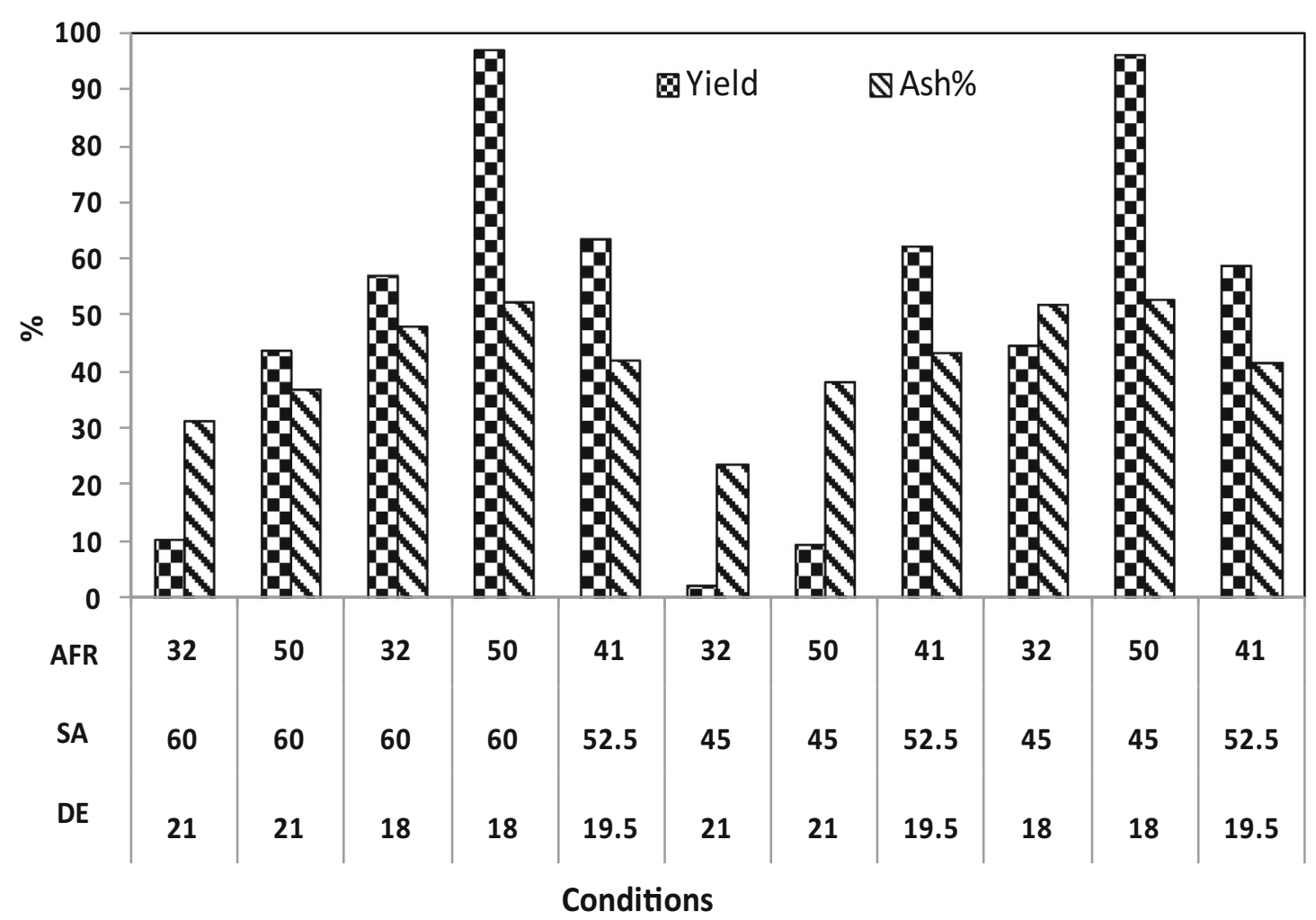

Fig. 5 Separation behavior at different conditions

\subsubsection{Physics behind segregation of particles on deck}

When the air current is blown through packed bed of solid materials it dilates the bed and produces a pressure drop due to drag force. When this drag force is equal to bed weight, particles behave like a fluid. The phenomenon is called fluidization. The manner of fluidisation depends upon airflow rate and proportion of fines present in feed. The fluid acts as pseudo-pneumatic fluid which segregate particles based on their densities. Particles having lower density report to the upper layer and particles with higher density move to the bottom of the bed. The heavier particles come in contact with the deck surface and acted upon by frictional force and vibrational force provided to the deck of table. The lighter particles at the upper layer are not affected by these two forces. Under combined effect of fluidisation, net gravitational force due to longitudinal angle of deck and vibrational force, particles move along the deck surface from the feed end towards the discharge end following the different trajectories and also by the resultant force arising out of all the forces acting on the particles. These are the frictional and vibrational forces due to the deck movement, drag force due to upward air flow and gravitational force for the heavier particle, while there is only effective gravitational force for the lighter particle. All these forces are associated with the particle movement. By the action of air flow, heavier particles fall onto the deck surface and come in contact with deck surface whereas lighter particles migrate to the upper layer. This phenomenon is called vertical segregation of particle (Fig. 6a). As heavier particle (Fig. 6b) comes in contact with surface, it is acted upon by vibrational force and moves towards upslope. Lighter particles move down slope along the shorter route by net gravitational force mainly as a result of side tilt and displacement caused by new feed entering the table. This separation of heavier and lighter particles is called horizontal segregation (Fig. 6b). Both horizontal and vertical segregation is presented pictorially in Fig. 6.

\subsubsection{Forces acting on the particles on deck}

Referring to the Fig. 3, when the transverse and longitudinal angles of the separating deck are in a position of $\beta$ and $\gamma$ respectively, particles move with the resultant angle $(\theta)$ of $\beta$ and $\gamma$. Forces acting on both heavier and lighter particles have been shown in Fig. 7

Let, $m=$ mass of the particle, $m g=$ gravitational force due to mass of the particle, $F_{\mathrm{N}}=$ normal force due to weight of the particle

Gravitational force $(\mathrm{mg})$ is resolved into two vectors,

(a) $m g \sin \theta=$ Force acting in the direction of deck surface 


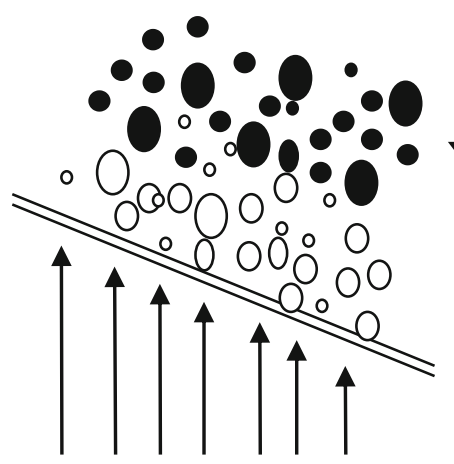

Vertical segregation due to air current

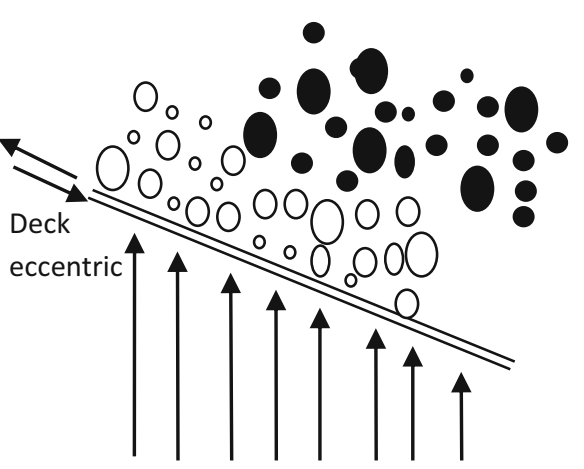

Horizontal segregation due to combined effect of Vibrational force and frictional force
Lighter particle

O Heavier particle

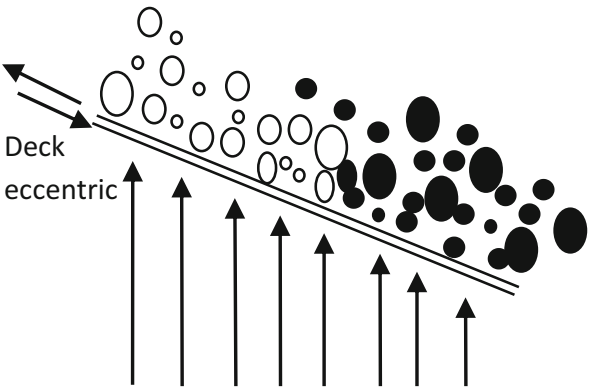

Ultimate separation due to both vertical and horizontal segregation

Fig. 6 Mechanism of segregation due to the combined effect of deck eccentric and airflow rate

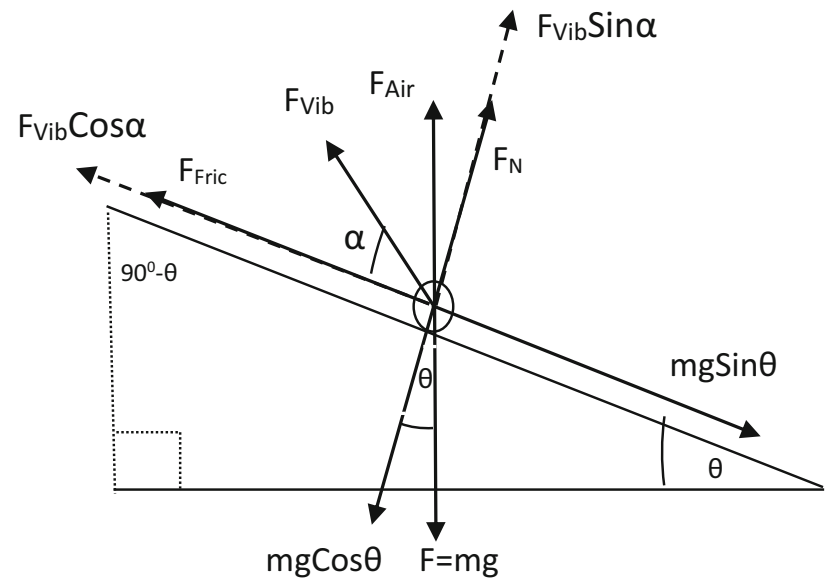

(a) For heavier Particle

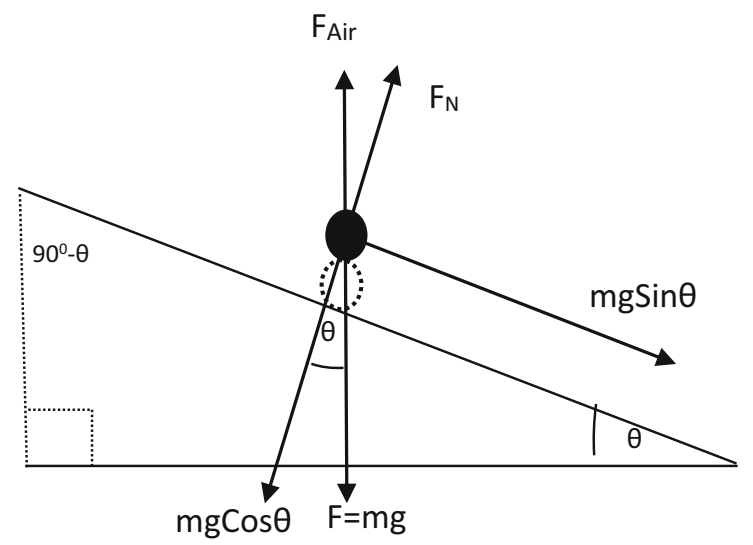

(b) For lighter particle

Fig. 7 Forces acting on the particles on the moving table (b) $m g \cos \theta=$ Force acting in the opposite to the normal force $\left(F_{\mathrm{N}}\right)$

$F_{\text {air }}=$ drag force due to air blown through porous bed

$F_{\text {vib }}=$ vibrational force due to deck eccentric

$F_{\text {fric }}=$ frictional force in the direction of opposite to the motion $=\mu F_{\mathrm{N}}=\mu m g \cos \theta$

$\alpha=$ angle between vibrational force and frictional force

Now, net force acting on lighter particle $F_{\text {net }}=F_{\text {accln }}=$ $\operatorname{mgsin} \theta+$ force due to new particle entering

Net force acting on heavier particle $F_{\text {net }}=F_{\text {net }}=$ $\mathrm{mgsin} \theta-F_{\text {fric }}-F_{\text {vib }}+F_{\text {air }}$

\subsection{Effect of variables on response}

The effect of single process variable in isolation cannot be studied as the process variables are inter-dependent. The combined effects are causing the effective separation. The contribution of individual and combined effect listed from the model is shown in Table 5. It seems that the contribution of the deck eccentric on yield is more than $56 \%$

Table 5 Variables contributing towards the responses

\begin{tabular}{llc}
\hline Variable parameters & \multicolumn{2}{l}{ Contribution $(\%)$} \\
\cline { 2 - 3 } & Yield & Ash \\
\hline A-deck eccentric & 56.2 & 62.7 \\
B-airflow rate & 29.2 & 22.0 \\
C-side angle & 10.32 & 10.56 \\
AB & - & 2.26 \\
BC & - & 1.62
\end{tabular}




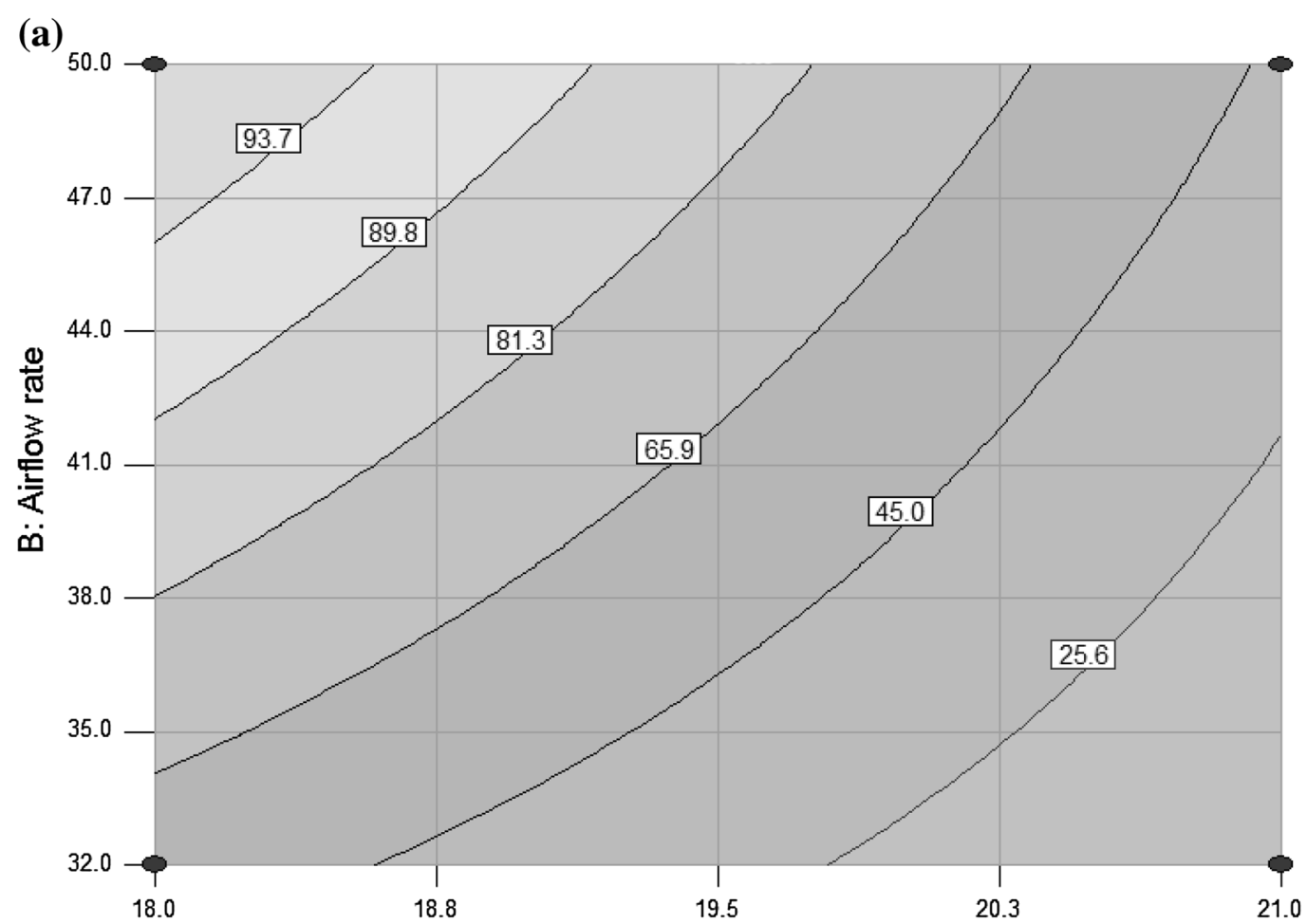

A: Deck Eccentric

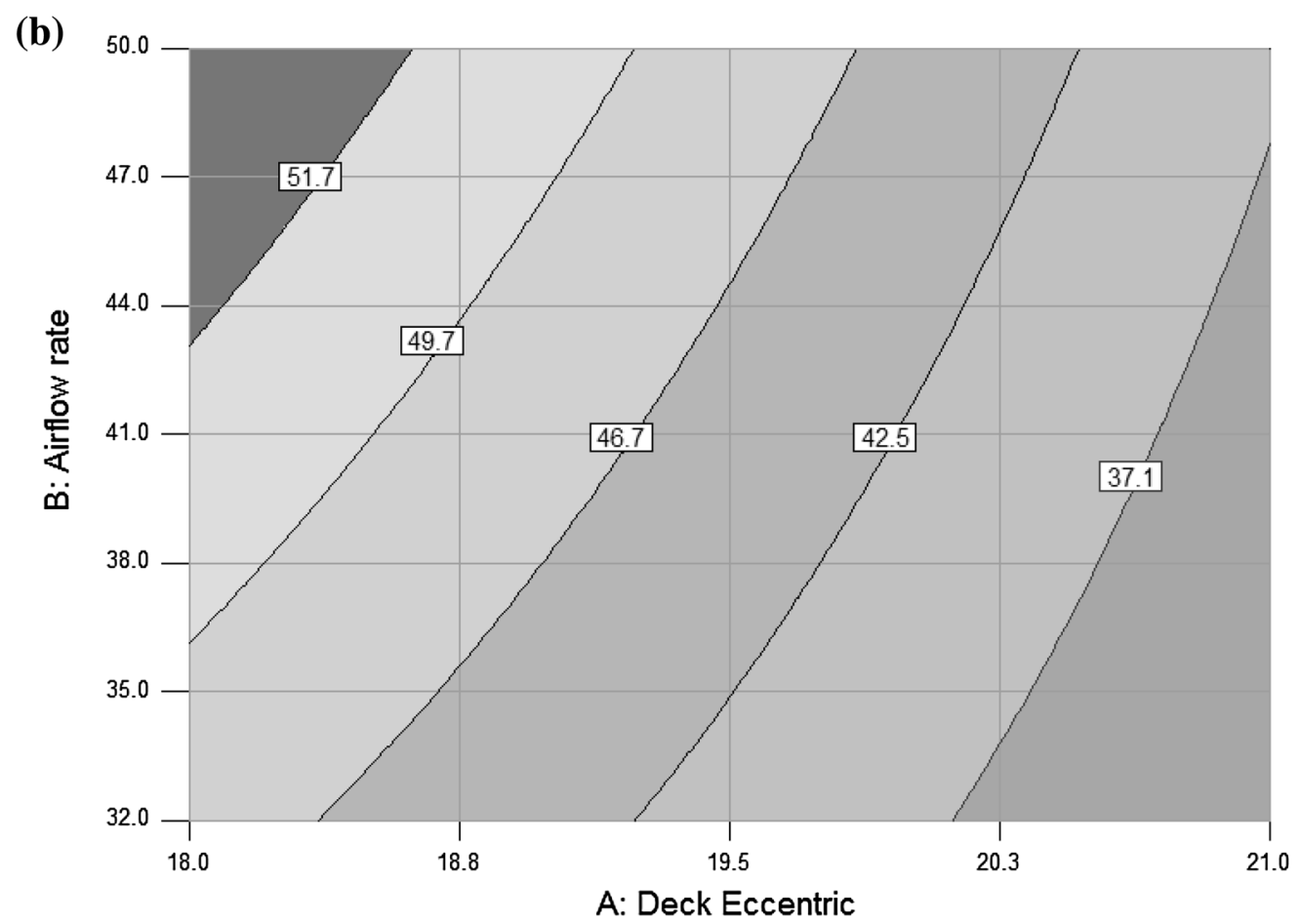

Fig. 8 Interaction effect of deck eccentric and airflow rate at $60 \mathrm{~mm}$ Transverse angle: aYield and $\mathbf{b}$ ash

followed by air flow rate and transverse angle. Similarly for the ash of the clean coal, within the design domain, effect due to deck eccentric is $62.7 \%$. The influences of the variables are discussed in the subsequent section.

\subsubsection{Interactive effect of deck eccentric and airflow}

The vibratory motion of the deck spreads the bed of particles on the deck. As the deck eccentric increases it allows 


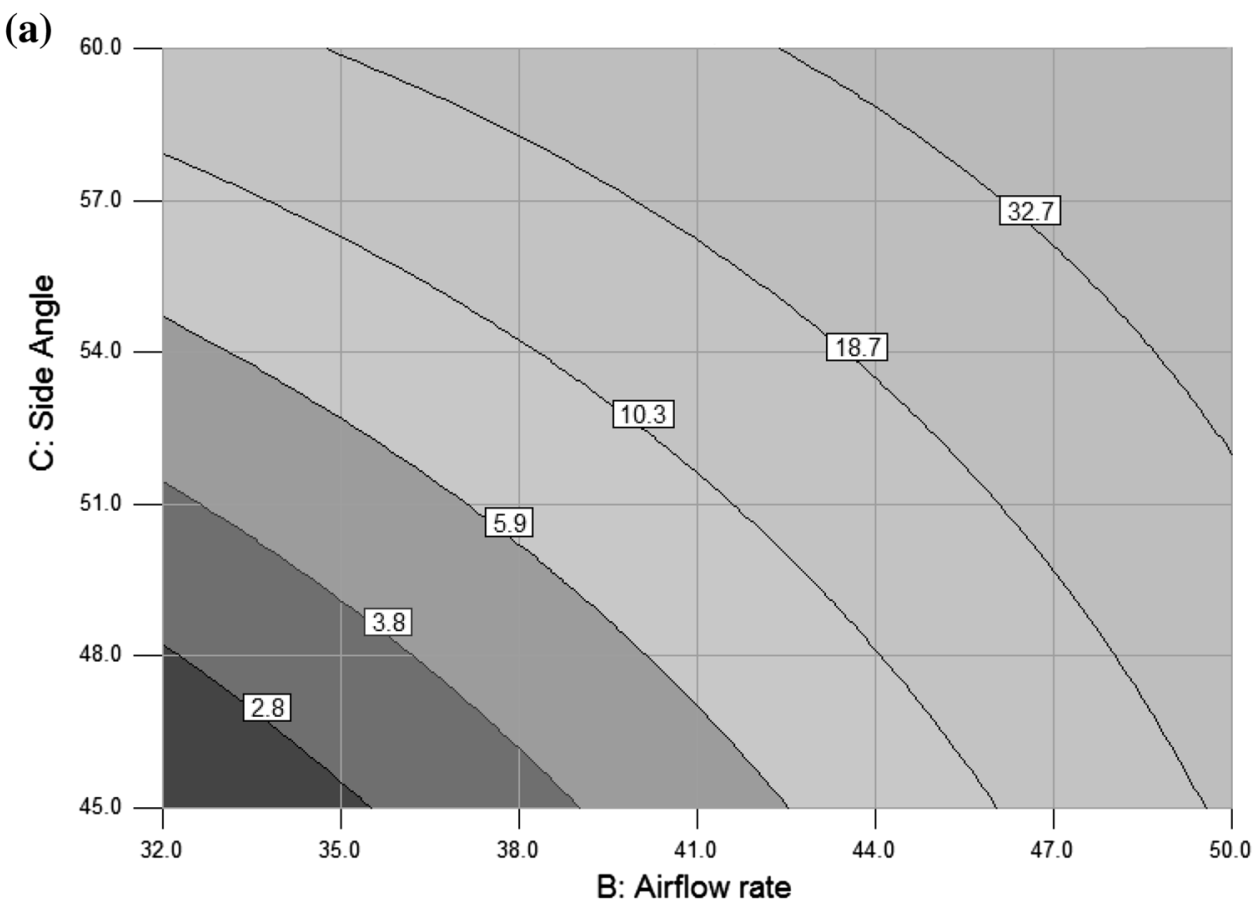

(b)

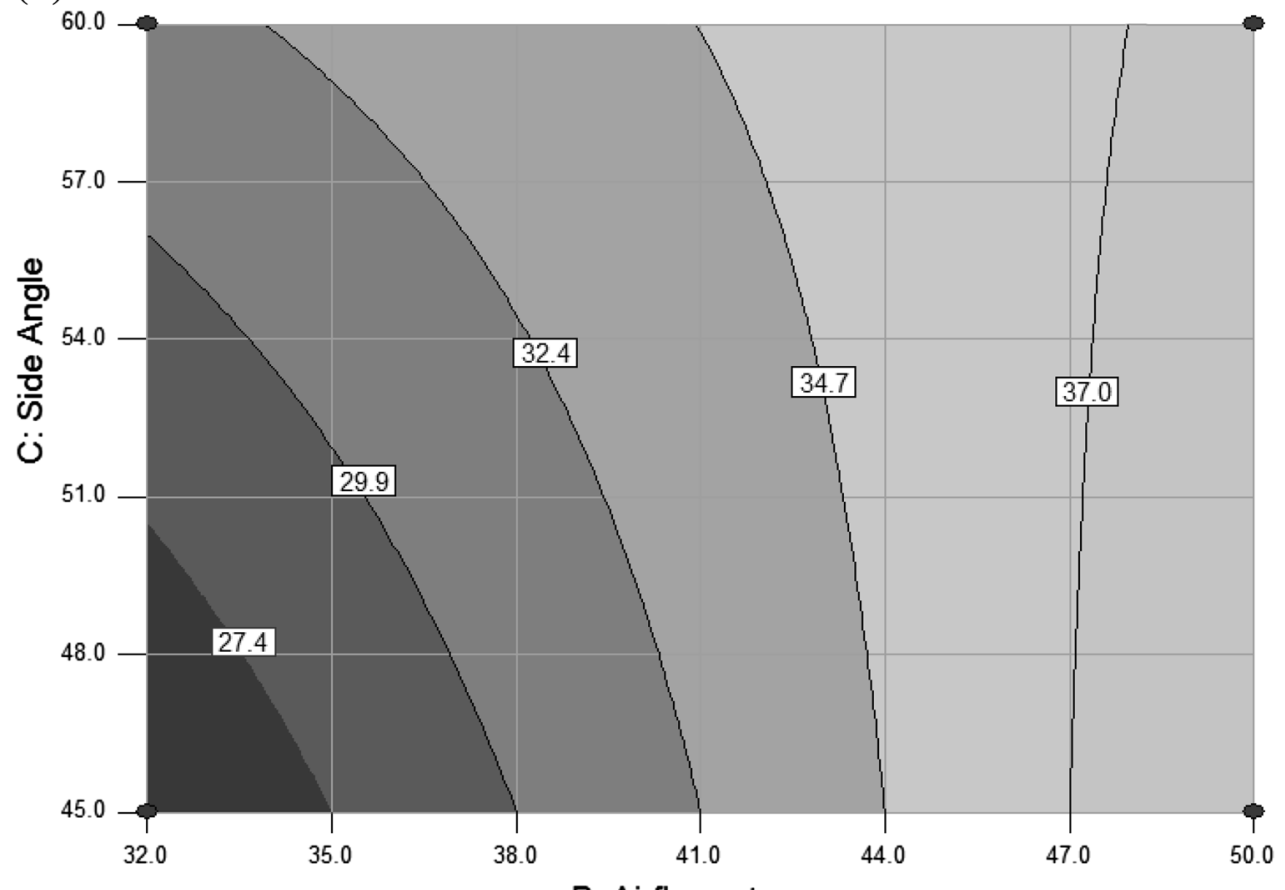

B: Airflow rate

Fig. 9 Interaction effect of Transverse angle and Airflow rate on ash of clean coal at $21 \mathrm{~Hz}$

the heavy particles to move towards the up slope of deck. The effect of deck eccentric was found to be dominant. This reflects that slight changes in deck vibration cause significant effect on the yield and also on the ash (Fig. 8a, b). The vibrating force acting on the particles has major contribution in the separation process. Combined effect of deck eccentric and airflow rate confers the separation of the heavier particles from coal sample. When there is only drag force of airflow, finer particles are blown into the upper layer (Fig. 6). In that case, feed material fails to undergo stratification according to the density difference. The contour Fig. 8 obtained from ANOVA indicates that the 


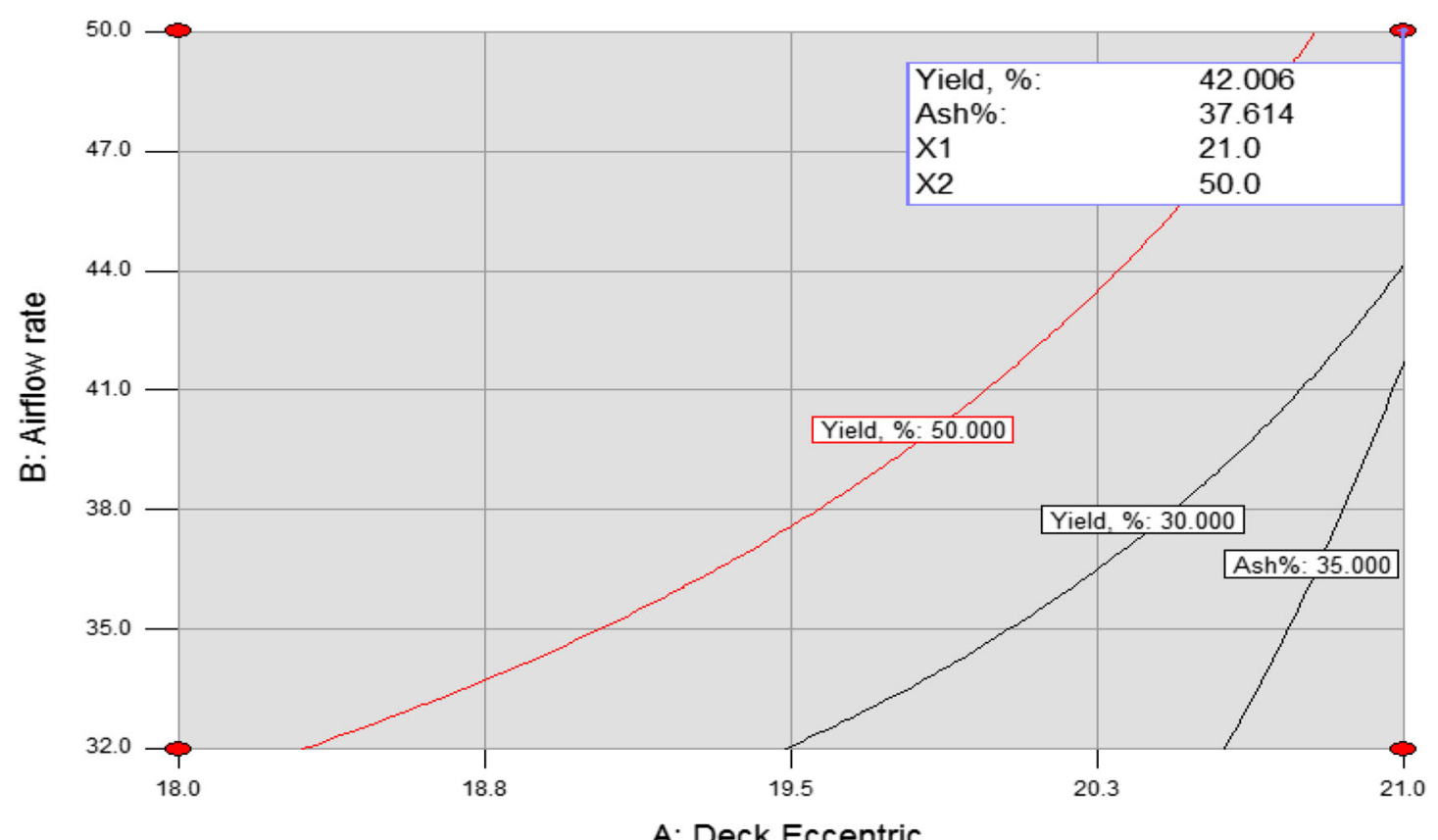

Fig. 10 Optimization with respect to deck eccentric and airflow rate

lower deck eccentric with higher airflow rate does not give effective separation, resulting high yield with high ash of about $51 \%$. At deck eccentric of $20 \mathrm{~Hz}$ and high air flow rate of $47 \mathrm{rpm}$, yield recovered is $68 \%$ with $45 \%$ ash. Figure 8 shows that clean coal below 37\% ash level is difficult to achieve in a single stage operation.

\subsubsection{Effect of airflow rate and transverse angle}

The increase in transverse angle and air flow rate of the deck decides the yield and grade of the clean coal (Fig. 9a, b). The increased transverse angle results in dominant gravitational force on particle. So flow of the material towards the lower side of the deck increases the ash of lighter fraction (clean coal). The heavier particles possessing high ash bearing minerals also have a tendency to report towards the lower end of the deck. Hence the effective reduction of ash does not occur. When the side tilt is decreased, more material moves towards the higher end of the deck. At air flow rate of $32 \mathrm{rpm}$ and transverse angle of $45 \mathrm{~mm}$, recovered yield is only $2.7 \%$ with $25.7 \%$ ash. However, with increase of air flow rate to $50 \mathrm{rpm}$ and transverse angle at $60 \mathrm{~mm}$, yield increases to $43.5 \%$ with $38.4 \%$ ash (condition 2 in Fig. 5). Due to the increase of air flow rate, drag force on the particle increases. According to the second law of thermodynamics, any system will show a tendency to achieve minimum i.e. reduction in free energy. The potential energy of the bed decreases when the bed is dilated. The stratification is a free energy reduction process completed through the redistribution and rearrangement of the particles.

\subsection{Optimization of operating conditions}

The optimization of the process parameters was carried out at different conditions with the help of a model formed by design expert software. From surface plot (Fig. 10) it is found that no significant clean coal could be obtained at $35 \%$ ash level. However, when the deck eccentric was reduced from 19.5 to $18.3 \mathrm{~Hz}$ and the air flow rate increased to $44 \mathrm{rpm}$, yield of clean coal increases from $30 \%$ to $50 \%$ with the increase of ash in clean coal. About $20 \%$ yield could be obtained at $35 \%$ ash. The organic efficiency is only 35 . Figure 10 shows also that at $21 \mathrm{~Hz}$ deck eccentric, $50 \mathrm{rpm}$ air flow rate and $60 \mathrm{~mm}$ transverse angle, concentrate of $42 \%$ with $37.6 \%$ ash could be recovered. The yields of clean coal at $35 \%$ and $37 \%$ ash level obtained from washability study and Air table are compared in Table 6.

Table 6 Separation performance at different ash levels for single stage operation

\begin{tabular}{lll}
\hline Ash level (\%) & 35 & 37 \\
\hline Washability (yield \%) & 57.2 & 60.0 \\
Pneumatic table (yield \%) & 20.0 & 42.5 \\
Organic efficiency & 35.0 & 71.0 \\
\hline
\end{tabular}


Table 7 Separation performance in two stages

\begin{tabular}{llllll}
\hline Conditions DE-SA-AFR & Ash $(\%)$ & Yield $(\%)$ & RC $(\%)$ & OE $(\%)$ & SE $(\%)$ \\
\hline Stage 1: $19.5-50-50$ & 45.6 & 84.4 & 95.7 & 96 & 47.7 \\
Stage 2: $20-40-50$ & 35.4 & 48.6 & 70.8 & 88.6 & 87.11 \\
\hline
\end{tabular}

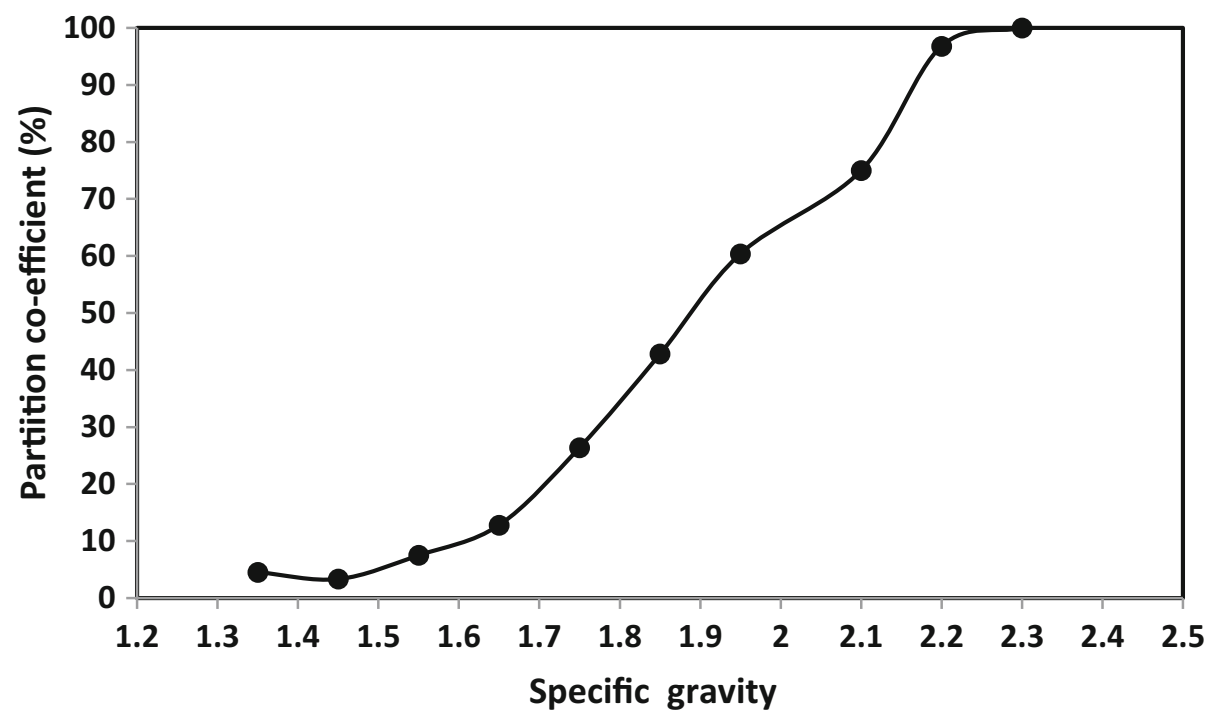

Fig. 11 Partition curve of the separation efficiency

\subsection{Multi stage processing}

From Figs. 8 and 9, it was found that clean coal with significant yield at 34\%-35\% ash level could not be achieved in single stage operation. Therefore, there is a need for two stages cleaning, where all the possible mineral matter associated with coal sample could be rejected in the first stage and the rougher concentrate would be reprocessed in the second stage for recovering the final concentrate.

From the experimental values (Fig. 5), it was found that condition $19.5 \mathrm{~Hz}, 50 \mathrm{~mm}, 50 \mathrm{rpm}$ for DE- SA- AFR respectively was selected for first stage separation and produces $84.4 \%$ yield with $45.6 \%$ ash content. The combustible in the reject stream is only $3.7 \%$. The concentrate of first stage was fed to the vibratory deck for second stage cleaning at 20-40-50 (DE-SA-AFR) condition. The overall yield of $48.6 \%$ could be achieved with ash of $35.4 \%$. Separation efficiency and organic efficiency are shown in Table 7 and higher separation efficiency was found in second stage. The partition coefficient represents the weight fraction of coal particle report to the reject stream. The value of probable error E could be calculated as $(\rho 75-\rho 25) / 2$. The coal particles with densities of $\rho 25$, $\rho 50$ and $\rho 75$ have the mass fractions of 25,50 and $75 \%$, respectively. The $\rho 50$ represents the deshaling density in the experiment. The $E_{\mathrm{p}}$ of the separation process is 0.18 (Fig. 11).

\section{Conclusions}

(1) The coal fines having high ash could be processed effectively by air fluidization technique and the combustible matters could be enriched significantly.

(2) Drag force due to air velocity and vibratory motion of the deck eccentric play significant role in segregation and stratification of the particles. Increased air velocity (32-50 rpm) plays important role in reducing the ash in clean coal.

(3) Increased deck eccentric had negative effect on the recovery of clean coal yield comparing with other variable parameters.

(4) In a single stage processing, ash level could be reduced to $37 \%$ with a yield of $42 \%$. However, there is a loss of combustibles in reject stream and organic efficiency of separation is $71.7 \%$.

(5) Multistage processing brought a significant improvement in separation. It improves the clean coal yield to $48.6 \%$ with $70.8 \%$ combustibles and ash content could be reduced from $54.5 \%$ to $35 \%$. The organic efficiency increases from $71.7 \%$ to $88.6 \%$. The value 
of probable error of 0.317 is an indicative of a favourable separation performance in a vibratory deck separator.

(6) Heat value of clean coal could be increased by three fold $(15,690 \mathrm{~kJ} / \mathrm{kg})$ of the feed sample. The processed coal sample would be useful for the boiler of thermal power plant and would increase the efficiency.

Acknowledgements The authors would like to express their sincere gratitude to Council of Scientific and Industrial Research, India for supporting the Mission mode net work project on dry beneficiation of Indian thermal coal (Project: ESC 0109).

Open Access This article is distributed under the terms of the Creative Commons Attribution 4.0 International License (http://crea tivecommons.org/licenses/by/4.0/), which permits unrestricted use, distribution, and reproduction in any medium, provided you give appropriate credit to the original author(s) and the source, provide a link to the Creative Commons license, and indicate if changes were made.

\section{References}

Burt RO (1984) Gravity concentration technology. Elsevier, Amsterdam, pp 352-372

Design-Expert ${ }^{\circledR}$ Software Version 8 -Stat-Ease. http://www.statease. com/soft-ftp

Dodbiba G, Sadaki J, Okaya K, Shibayama A, Fujita T (2005) The use of air tabling and triboelectric separation for separating a mixture of three plastics. Miner Eng 18:1350-1360

Geological survey of India (GSI) (2014), http://coal.nic.in/content/ coal-reserves, accessed 23rd November 2016
Gupta N, Bratton R, Luttrell GH, Ghosh T, Honakar RQ (2012) Application of air-table technologies for cleaning Indian coals. In: Young CA, Luttrell GH (eds) Separation of technologies for minerals, coal, and earth resources. Society for Mining, Metallurgy, \& Exploration, Englewood, pp 199-209

Haider A, Levenspiel O (1989) Drag co-efficient and terminal velocity, of spherical and non-spherical particles. Powder Technol 58(1):63-70

Honaker RQ, Sracoglu M, Thompson E, Bratton R, Luttrell GH, Richardson V (2008) Upgrading coal using a pneumatic density based separator. Int J Coal Prep Utili 28(1):51-67

IS 1350-1: Methods of test for coal and coke, Part I: Proximate Analysis, 1984

IS 9127-5: Methods for the petrographic. Analysis of bituminous coal and anthracite, Part-5, 2004

Li HB, Luo ZF, Zhao YM, Wu WC, Zhang CY, Dai NN (2011) Cleaning of South African coal using a compound dry cleaning apparatus. Min Sci Technol 21(1):117-121

Lockhart NC (1984) Review Paper: dry Beneficiation of Coal. Powder Technol 40:17-42

Ministry of Environment and forest, Gazette Notification (GSR 560(E) and 378(E), 1998

Osborne DG (1988) Pneumatic separation, coal preparation technology. Graham and Trotman, Norwell, pp 373-386

Patil DP, Parekh BK (2011) Beneficiation of fine coal using the air table. Int J Coal Prep Utili 31(3-4):203-222

Shobhana D, Gangadhar B, Gopalkrishna SJ (2015) Amenability to dry processing of high ash thermal coal using a pneumatic table. Inter J Min Sci Technol 25:955-961

Yang Xuliang, Zhao Yuemin, Luo Zhenfu, Song Shulei, Duan Chenlong, Dong Liang (2013) Fine coal dry cleaning using a vibrated gas-fluidized bed. Fuel Process Technol 106:338-343

Zhao ZF, Chen YM, Chen QR, Fan MM, Tao XX (2002) Separation characteristics for fine coal of the magnetically fluidized bed. Fuel Process Technol 79(1):63-69

Zhao PF, Zhao YM, Chen ZQ, Luo ZF (2015) Dry cleaning of fine lignite in a vibrated gas-fluidized bed: segregation characteristics. Fuel 142:274-282 